\title{
Reflexiones sobre sexualidad, reproducción y paternidad en estudiantes universitarios en México
}

Reflections on sexuality, reproduction, and fatherhood in university students of Mexico

\section{María Alejandra Salguero Velázquez}

Doctora en Sociología. Profesora Titular de Psicología. Facultad de Estudios Superiores Iztacala. Universidad Nacional Autónoma de México.

alevs@unam.mx

\author{
María J. Marco Macarro \\ Doctora en Psicología. Profesora Contratada, Departamento de Antropología Social, Psicología Básica y Salud Pública. \\ Universidad Pablo de Olavide de Sevilla (España) \\ mmarmac@upo.es
}

\section{CUERPOS, SEXUALIDADES Y PODER \\ MONOGRÁFICO COORDINADO POR JOSÉ MARÍA VALCUENDE. Universidad Pablo de Olavide (Sevilla).}

\begin{abstract}
RESUMEN
La sexualidad y paternidad, incorporan ideologías, normas, prácticas y significados diferenciales en escenarios socio-culturales. Instituciones heteronormativas como la familia y escuela no los consideran. Sin embargo, son eventos que viven los jóvenes, en ocasiones sin uso de anticoncepción, interrumpiendo su trayectoria académica por embarazos no planeados. El objetivo de la investigación es analizar la vivencia de la sexualidad, reproducción y paternidad en estudiantes universitarios. Se incorporan datos de entrevistas a profundidad con dos estudiantes de psicología en la FESI, México, que asumieron la paternidad ante un embarazo no planeado. Se identifican prácticas y discursos de poder de género y sexual en torno a su vida sexual, uso de anticoncepción y embarazo en función de la relación de pareja, formal o informal. La noticia del embarazo los confronta con la familia, llevándolos a negociar la continuidad como estudiante, pareja, padre, trabajador.
\end{abstract}

\section{ABSTRAC T}

Sexuality and fatherhood incorporate ideologies, norms, practices, and meanings from different socio-cultural settings. Heteronormative institutions such as family and the school do not consider the experience of sexuality and parenthood over the course of university studies; however, some students interrupt their studies for unplanned pregnancies. The objective is to analyse experiences of sexuality, reproduction, and fatherhood in university students. The data incorporate in-depth interviews with two Mexican students of psychology at FESI who assumed the fatherhood to an unplanned pregnancy. Motives, practices, and discourses of gender and sexual power about their sexuality, the use of contraception, and pregnancy were analyzed in terms of the relationship, whether formal or informal. The news of pregnancy is presented to the family, impelling them to negotiate continuity as a student, partner, parent, and worker.

PALABRAS CLAVE

jóvenes | sexualidad | reproducción | masculinidad | paternidad

KEYWO RDS

youth | sexuality | reproduction | masculinity | fatherhood

\section{Introducción (1)}

Desde el feminismo se ha planteado la desnaturalización e historización de la sexualidad, ya que con base en el sistema de organización sexo-genérica (Rubin 1997), se incorporan una serie de dispositivos de poder que establecen ideologías, valores, normas, sanciones, prácticas y significados diferenciales desde los que se construyen cuerpos, espacios y tiempos (Foucault 1988). Un caso particular es la sexualidad y reproducción en las y los jóvenes universitarios, pues desde la normatividad institucional de la familia y la escuela "eso no se contempla, ni se considera", ellos y ellas ingresan a la universidad para estudiar y terminar una carrera universitaria, bajo el supuesto de que "cuando terminen ya habrá tiempo para establecer relaciones amorosas e incorporar la sexualidad". Sin embargo, los datos de la Encuesta Nacional en Salud 2012 señalan que el $55.3 \%$ de los jóvenes tienen actividad sexual, de los cuales un $23 \%$ declara haber iniciado entre los 15 y 19 años, siendo los hombres quienes comienzan su vida sexual antes 
que las mujeres. González, Rojas, Hernández y Olaiz (2005), en su estudio llevado a cabo con estudiantes de nivel medio en escuelas técnicas de la ciudad de México, indican que hay un aumento en el porcentaje de inicio de la vida sexual en los jóvenes de 18 a 19 años. Ibáñez (1998), analizando el tipo de pareja sexual en jóvenes mexicanos, encontró que la mayoría de las jóvenes refiere haber tenido relaciones sexuales con su novio y en el caso de los hombres se encuentra una mayor variación en el tipo de relación de pareja. En la encuesta realizada con estudiantes del Distrito Federal en la ciudad de México, Fleiz y otros (1999) indican que el $74.8 \%$ de los adolescentes que habían iniciado su vida sexual utilizaron algún método anticonceptivo, mientras que el $25.2 \%$ no usó protección alguna. El método más usado tanto para hombres como para mujeres fue el condón (60.9\%), aunque el $30.1 \%$ de los jóvenes no utilizaron, lo cual incrementa la probabilidad de que ocurran embarazos no planeados, abortos, infecciones de transmisión sexual y VIH. Particularmente en México, hay poca investigación con estudiantes universitarios en comparación con los de otros niveles educativos. Es necesario aproximarnos cualitativamente a conocer la vida cotidiana de los y las estudiantes dentro de las instituciones de educación superior, como personas que participan en una diversidad de prácticas en contextos socioculturales aparte de la escuela, donde establecen una relación diversa, en ocasiones conflictiva o contradictoria. Un caso particular es la manera cómo se vive y significa la sexualidad, la reproducción y la paternidad.

\subsection{Sexualidad y reproducción en jóvenes varones}

Analizar los significados y vivencia de la sexualidad, particularmente en los jóvenes varones, resulta importante, pues existe un discurso social que plantea como problemática social el poco involucramiento en prácticas de cuidado del cuerpo y uso de anticoncepción, lo cual lleva a algunos jóvenes a una paternidad no planeada, enfrentándose a procesos complicados de toma de decisiones donde tendrán que valorar entre la continuidad de la trayectoria académica, la formalización de la relación de pareja y familia, el conseguir un trabajo para cumplir con la responsabilidad de proveeduría, y la construcción de identidad como padre.

Acercarnos a la mirada de los jóvenes varones permitirá cuestionar categorías hegemónicas en torno a la sexualidad y reproducción, identificando formas que no responden necesariamente a los modelos dominantes y cuya significación ha sido forzada en función de esquemas de conocimiento que dicotomizan realidades plurales y complejas. Existe un discurso dominante desde las instituciones que reprime y sanciona la sexualidad, el deseo, el goce y el placer en los jóvenes (Menkes 2004, Szasz 1997), ya que la vivencia de la sexualidad ha sido históricamente reglamentada. Foucault (1987a) plantea que se encuentra relacionada con discursos, significados y prácticas sociales, de manera que la puesta en discurso del sexo desde el siglo XVII se convirtió en una regla a través de complejos dispositivos de poder y control desde las instituciones. La sexualidad se convierte en un problema económico y político desde donde se analiza la edad del matrimonio, la precocidad y frecuencia de las relaciones sexuales, la manera de tornarlas fecundas o estériles, la incidencia de las prácticas anticonceptivas. Un aspecto fundamental es el papel de las instituciones que, a través de discursos y prácticas reglamentadas, "sugieren, dan opiniones y consejos" sobre cómo pensar, sentir, comportarse, cómo luchar contra los deseos y placeres, cómo regularlos, controlarlos y dominarlos para que no interfieran con los tiempos normados y reglamentados. Se construye un discurso en torno a la "austeridad sexual", el cual se relaciona con experiencias y formas de relación con el cuerpo y la salud. La virtud sexual para el caso de las mujeres se centrará en la pureza, virginidad y fidelidad, en tanto que para el hombre será la concepción del dominio como libertad lo que afirmará el carácter viril. La elaboración de sí como sujeto moral consistirá en instaurar la estructura de virilidad a través del control y dominio hacia los demás en la práctica sexual (Foucault 1988). La regulación y dominio de los placeres asegura un uso de adaptación a las necesidades, momentos y circunstancias sobre lo que se debería desear, la manera que hay que desearlo y en qué circunstancia. Habrá que luchar, mandar y dominar los deseos y los placeres a través de las prácticas de sí (Foucault 1987b). Es así que la sexualidad sigue siendo un terreno de conflicto y cuestionamiento, predominando una visión reduccionista y determinista donde la complejidad de la experiencia se reduce a impulsos, instintos, genes, hormonas o incluso procesos inconscientes.

A su vez, la sexualidad y la reproducción se han visto influenciadas por el sistema de organización genérico.

Las mujeres serán consideradas como carentes de deseo sexual, en tanto que, para los hombres, la sexualidad, tener sexo y desear relaciones sexuales son signos constitutivos de la identidad masculina, formando parte de un proceso de aprendizaje complejo que se inicia con la institución familiar, en la infancia, adolescencia y continúa a lo largo de la trayectoria de vida (Seidler 2000, Kaufman 1994). 
Aprenden que deben ser los que guíen, decidan cómo y cuándo vivir su sexualidad, que pueden vivir diferentes tipos de deseo sexual con las mujeres, las novias que llevarán al matrimonio y se convertirán en esposas, tendrán hijos y se apropiarán de su trabajo convirtiéndolos en eternos proveedores (Núñez 2007, Vendrell 2010). El vínculo sexual con sus esposas no se limita a la voluntad ganada por el trabajo, sino también a la voluntad de ellas de tener hijos, de volverse madres, lo que conforme a su perspectiva es el deseo de las mujeres. Amuchástegui (2007) investigó sobre la forma en que algunos hombres mexicanos se construyen a sí mismos como sujetos de deseo sexual, de placer, de procreación y de paternidad. Integró narraciones autobiográficas de hombres jóvenes, encontrando que afirman y se reconocen como sujetos de una sexualidad activa, exclusivamente heterosexual con múltiples parejas. En el caso de los entrevistados, una vez que ocurre el embarazo, inexorablemente se suceden el matrimonio y la división sexual del trabajo, y con ello la percepción de las mujeres como carentes de deseo sexual. Señala que en la cultura sexual dominante en México, las mujeres tienen que trabajar activamente para separar la sexualidad de la reproducción, mientras que para los hombres están "naturalmente" separadas y por tanto son un asunto de elección. El erotismo y la sexualidad se subordinan a la exaltación de la reproducción.

Es importante abordar las relaciones de género, construcciones y estereotipos culturales en la configuración de la sexualidad, reproducción y embarazo, lo que significa ser madre o padre. Las barreras familiares, institucionales y sociales que impiden vivir una sexualidad placentera incorporando una ética del cuidado, o incluso en aquéllos jóvenes que han decidido ser padres involucrados y responsables, o quienes han finalizado su relación con la madre del niño, o no quieren jugar un papel activo como padres (Stern 2004). Un evento como el embarazo y la paternidad los lleva a re-significar su identidad.

\subsection{Reproducción: paternidad en jóvenes}

La investigación sobre paternidad se ha incorporado como parte de la agenda en los estudios feministas de género, de las masculinidades, de la sociología de la familia, y de las políticas públicas. Los significados sobre las relaciones genéricas y formas de vida entre mujeres y hombres han tenido cambios importantes a partir de las transformaciones sociales y culturales como el cuestionamiento de las desigualdades de género y los derechos de las mujeres planteados por el movimiento feminista y la Conferencia Mundial de la mujer en 1975 (Chant y Craske 2003, Amuchástegui 2001, Salles y Tuirán 1998).

Las prácticas y significados bajo los cuales se vive y ejerce la paternidad integran aspectos relacionados con el poder genérico en la relación de pareja. Esto incluye el deseo por los hijos y la manera en que éstos se insertan en el proyecto de vida. Algunas investigaciones en Latinoamérica sobre paternidad como las de Doria, Oliveira y Muzskat (1999), Fuller (2000) y Viveros (2000) encuentran que para los varones es un proceso de transformación de identidad, de cambio a un nuevo período de vida que es la adultez, una responsabilidad que implica la renuncia a su autonomía individual y un mayor compromiso tanto material como moral, representa la necesidad de establecer un vínculo con la pareja y los hijos.

En México, Nava (1996), Rojas (2000), Jiménez (2001) y Salguero (2002) coinciden en que ser padre es un hecho que cambia radicalmente la vida. Para algunos entrevistados la decisión de tener hijos forma parte del proyecto de vida que van construyendo con la pareja, significa una responsabilidad pero también es una experiencia emocional, de aprendizaje permanente y que disfrutan. Para Salguero y Pérez (2011a) es un proceso complejo y diverso que cambia y se transforma a partir de múltiples influencias como podrían ser el momento de conformación de la familia, los procesos de negociación que se gestan al interior de ella, la decisión de tener hijos, los requerimientos de la pareja. Haces (2006) investigó en hombres y mujeres del municipio del Valle de Chalco en la ciudad de México cómo se significa la paternidad, encontrado que en ocasiones hay confrontación de los varones con sus parejas por las exigencias de ellas de una mayor participación. Considera que en la generación de los jóvenes se comienza a cuestionar la figura tradicional del padre y la madre, observándose deslizamientos encaminados a transformar las figuras estereotipadas del padre y la madre, como lo han señalado Gutmann (2000) o Salguero y Pérez (2008) en investigaciones llevadas a cabo con varones en México.

De Jesús y Cabello (2011) investigaron la vivencia y significado de la paternidad en la trayectoria de vida de hombres jóvenes en el estado de Monterrey en México. Sus resultados muestran que el tipo de relación familiar se convierte en algo fundamental influyendo en el interés de los jóvenes para continuar sus estudios, lo cual se asoció con el embarazo de una pareja, llevándolos a "normalizar" el embarazo. Los datos coinciden con los de Stern (2004) en su investigación sobre sexualidad y reproducción en jóvenes mexicanos en el sentido de que no solo las condiciones socioculturales del contexto, sino las expectativas 
en la relación de pareja y la familia, lleva a los jóvenes a considerar como algo normal su embarazo. Los jóvenes con bajo nivel socioeconómico y de escolaridad son quienes mantienen relaciones sexuales y ante la inminencia de un embarazo se incorpora la paternidad. Algunos comentan que "no usan métodos anticonceptivos, porque si llegara un embarazo, se responsabilizarían", incorporan la formalización de la unión con la pareja y el ser padres como algo "normal y esperado". Ante la noticia del embarazo, la familia, los amigos y el contexto que les rodea terminan aceptándolo. Algunos dejan la escuela y tienen que buscar un trabajo que les permita cumplir con la obligación y responsabilidad de esa nueva etapa de vida.

Sin embargo, no se tiene información de que en estudiantes universitarios se considere normal la idea de un embarazo, ya que en ese momento de la trayectoria de vida se contemplaría el involucramiento en las actividades académicas, y no en procesos como el noviazgo, relaciones amorosas y la sexualidad, eso se dejaría para otro momento. La formación universitaria se sigue considerando la posibilidad de movilidad social ascendente, el recurso para alcanzar mejores condiciones de trabajo y un mayor disfrute de la vida en general (Testa y Sánchez 2003). De ahí que investigaciones sobre trabajo y familia hayan tenido gran importancia, pues permiten identificar las actividades familiares que hacen posible su reproducción cotidiana y generacional, y dentro de ello, el énfasis otorgado para que los hijos e hijas puedan tener y concluir una formación universitaria, considerando que les permitirá acceder a un mejor nivel de vida (Torrado 2006, Wainerman 2003).

Hasta hace relativamente poco, en México la idea imperante en torno a los estudiantes que cursan educación superior era que son jóvenes privilegiados en términos sociales, económicos y culturales. Comúnmente, la cobertura a nivel licenciatura se calcula en el rango etario de 19 a 23 años. Esto es asi porque se considera que tales edades son las "normales" para que los jóvenes estudien una licenciatura. Sin embargo, no se considera que en esta trayectoria se transite por eventos como el enamoramiento, relaciones de pareja, sexualidad, embarazo, maternidad y/o paternidad, lo cual va más allá de los criterios normativos, uniformes y administrativos de "lo deseado" en la trayectoria universitaria.

Nos enfrentamos a diversas y complejas formas de ser joven universitario. Ya no es solo producto de una prolongada construcción histórica social que la liga al paso por instituciones educativas, sino que adquiere una complejidad en el entramado de las relaciones sociales que establece en el mundo social en el que participa. En algunos casos, la prolongación de la esperanza de vida, combinada con oportunidades de continuar en niveles educativos superiores y con menores oportunidades de alcanzar un puesto de trabajo con ingresos acordes a la manutención propia, van configurando estas nuevas formas de habitar la juventud. Las cuales están relacionadas con el disfrute del tiempo libre, los consumos culturales y experimentación, es decir, con un tiempo liberado que no era socialmente permitido en otras generaciones. Los nuevos estilos de vida se han extendido sobre todo en los países de mayor desarrollo económico y alcance de la seguridad social, pero también aparecen como fenómenos emergentes en los países latinoamericanos. Se dan de forma paralela otros modos de organización del ciclo vital en donde se produce una especie de intersección entre las actividades propias a la condición juvenil como la conjunción entre maternidad/paternidad temprana, la continuidad educativa y el consumo de bienes propios a la cultura juvenil: recitales, salidas nocturnas, entre otras formas de habitar la juventud que en algunas ocasiones adquieren la denominación de trayectorias atípicas o también fallidas.

El que se considere la paternidad como un evento que trastoca la trayectoria académica, llegando a considerarla fallida, tiene que ver con la normatividad de las prácticas sociales, las cuales incluyen criterios, procedimientos y regulaciones de edad, particularmente la paternidad se considera como posible en ciertos momentos, generalmente cuando los hombres son adultos, han terminado una carrera profesional y formalizan la relación de pareja. Nada más complejo que eso...

Reconocer la diversidad de formas de habitar la juventud y prácticas de paternidad, requiere de un enfoque detallado de las diferencias individuales, actividades, compromisos y recursos disponibles, lo cual va conformando la trayectoria de vida de un joven y su construcción de identidad como padre (Salguero y Pérez 2008).

Con base en lo anterior, nos interesa indagar cómo incorporan la vivencia de la sexualidad, el uso de anticoncepción y cómo afrontan la noticia de embarazo.

Esto no sólo no es obvio, sino que resulta mucho más complicado y diverso de lo que se podría imaginar. Brannen y Nielsen (2004) señalan que, en general, en la población estadounidense de generaciones 
anteriores, los hombres se mostraban menos dispuestos a negociar los cuidados infantiles bajo el argumento de que eso correspondía a las mujeres, en tanto que para algunos padres jóvenes la crianza de los hijos es algo compartido con la pareja, se involucran de manera directa en el cuidado y atención. Ser padre es un concepto con una gran carga de valor, está asociado a significados positivos como transmitir valores, encontrar un sentido de vida (Hoghughi 2004). Los conceptos valorativos forman parte de las nociones dominantes y expectativas sociales de lo que es "adecuado" para los padres, lo cual se refuerza en la literatura sobre consejos para la crianza (Walzer 2004), algo difícil ya que en la actualidad no es suficiente con ser padre, hay que ser "buen padre", tener paciencia, disponibilidad y estar atento a las necesidades de los hijos. El planteamiento anterior se vuelve más complejo cuando las expectativas de los jóvenes están dirigidas a terminar sus estudios universitarios y se enfrentan a un embarazo no planeado.

\section{Objetivos y metodología}

El presente trabajo tiene como objetivo analizar la vivencia de la sexualidad, reproducción y paternidad en la trayectoria de vida de estudiantes universitarios. Forma parte de proyecto más amplio sobre sexualidad, reproducción y paternidad en jóvenes de la Facultad de Estudios Superiores lztacala de la UNAM y de la Universidad Pablo de Olavide en Sevilla, España. Integra los ejes de reflexión de los proyectos "Significado y vivencia de la maternidad y la paternidad en la trayectoria de vida de estudiantes universitarios" (RN306813) y del Proyecto Iberoamericano para el Estudio Sociohistórico de las Sexualidades. Incorpora una metodología cualitativa, ya que permite estudiar a profundidad los fenómenos sociales, explorar la red de relaciones que forman parte de las significaciones, valores y prácticas de las mujeres y los hombres. Reconoce la complejidad de las estructuras sociales y el papel de agencia de los individuos en el proceso de construcción de identidad. Permite abordar y analizar la lógica de lo diferente, lo novedoso y lo "otro"; recupera lo cultural y el cuestionamiento del orden existente como serían los discursos y prácticas en torno a los estereotipos masculinos y la paternidad. En el caso que nos ocupa, posibilita identificar la complejidad de la práctica social en el proceso de construcción de trayectorias de vida en estudiantes universitarios, visualizando y señalando la extensa heterogeneidad y complejidad de las relaciones familiares, escolares, de amigos, pares, pareja, etc., en su proceso de formación como personas, en tanto formas de pensamiento, comportamiento y sentimiento.

La estrategia de investigación incorporó entrevistas a profundidad. En este trabajo se incluyen solamente los datos de dos estudiantes varones: Andrés y Javier, quienes cursaban el tercer semestre de la carrera de Psicología de la Facultad de Estudios Superiores Iztacala, ubicada en el Estado de México. Ambos de 24 años de edad, viviendo como hijos de familia en casa de sus padres, quienes asumían los gastos económicos durante su período escolar. El nivel socioeconómico de las familias es medio, les permite cubrir las necesidades de vivienda y transporte, pero también el asistir a algún concierto de música, fiestas con amigos, o alguna actividad de la cultura juvenil.

Se llevó a cabo el proceso de negociación de manera personal, invitándolos a participar en la investigación debido a la trayectoria académica que llevaban hasta antes del embarazo de la pareja, tenían un promedio de 8.50 en sus calificaciones y no adeudaban materias. Aceptaron la invitación para la conducción de entrevistas, las cuales se llevaron a cabo en un edificio de la Universidad. Se consideró pertinente a nivel metodológico que fueran otras jóvenes estudiantes quienes generaran el ejercicio reflexivo a manera de diálogo situado con los jóvenes entrevistados de la carrera de psicología. Andrade, Shedlin y Bonilla (1987) consideran la entrevista como parte integral del proceso de recolección de datos, ya que permite acceder a los pensamientos y sentimientos de los participantes sobre las actividades y los procesos que viven. Para Castro y Bronfman (1999) la información proporcionada suele ser el resultado de una elaboración de la persona más que una respuesta a una pregunta específica. A su vez, Giddens (1998) plantea el ejercicio reflexivo como parte del proceso de revaloración de significados de las prácticas de los individuos. En nuestro caso particular, sobre sus experiencias como estudiantes universitarios, como hijos de familia, parejas o padres, los dilemas y conflictos a los que se habían enfrentado y qué han hecho al respecto, profundizando en eventos y significados como la sexualidad, el embarazo y el ser padre en la trayectoria universitaria.

Respecto a la formación profesional, se decidió trabajar con estudiantes de la carrera de psicología porque, aun cuando se tiende a pensar que los campos disciplinarios son neutros respecto al género, cada disciplina promueve ciertas visiones del mundo que se relacionan con determinado modelo de concepción 
sobre la sexualidad, reproducción, discursos de género, lo cual se incorpora más en carreras de Ciencias Sociales.

Los ejes de análisis propuestos fueron: 1) vivencia de la sexualidad, 2) anticoncepción y noticia del embarazo, 3) identidad paterna. Nos interesa recuperar las experiencias y significados sobre las experiencias de los jóvenes universitarios respeto a su vivencia de la sexualidad, reproducción y paternidad sin pretender establecer generalizaciones, sino incorporar la reflexión compartida desde la voz de los participantes.

\section{Resultados}

Una vez llevadas a cabo las entrevistas, se procedió a la transcripción y análisis de datos. Una primera etapa correspondió a las múltiples lecturas para proceder a la codificación abierta, al análisis y fragmentación de la información para organizar el material. La segunda etapa implicó la organización de la información para identificar conceptos y elaborar categorías que nos permitieran contactar con la información teórica, y proceder al análisis de contenido e interpretación temática.

\subsection{Vivencia de la sexualidad}

En el caso de los estudiantes de Psicología entrevistados, el plan de estudios no contempla curricularmente asignaturas o cursos sobre sexualidad, lo cual resulta contraproducente en el proceso de formación universitaria. En la Universidad Nacional Autónoma de México se imparte educación pública de carácter laico; no obstante, el tema de la sexualidad no forma parte del currículo, se da por hecho que es un saber incorporado, aun cuando los y las jóvenes externen una serie de dudas y conflictos en sus relaciones interpersonales en relación a la sexualidad.

Institucionalmente se considera que las y los jóvenes han estado expuestos a procesos reflexivos en los ámbitos de sexualidad y reproducción en sus ámbitos familiares, lo cual como señalan los entrevistados difícilmente es abordado por los padres, madres, o familiares cercanos. Javier comenta: "en casa de mis padres no se habla de sexualidad, mis padres suponen que esos temas tendría que verlos en la escuela, más si estoy estudiando Psicología". Podemos ver cómo la institución educativa delega el saber y la reflexión sobre la sexualidad en la familia, y a su vez la familia lo delega a la escuela.

Andrés y Javier señalan que en ocasiones pueden hablar sobre la sexualidad en alguna asignatura clínica o educativa, pero de manera general, pues no forma parte de las temáticas de las asignaturas ni de los programas académicos. Sin embargo, el que estén cursando una licenciatura en Psicología, sí los coloca como estudiantes que han estado más expuestos a temas sobre igualdad y equidad de género, por lo menos a nivel discursivo la sexualidad sería visualizada como un ejercicio de derechos, que debería ser placentera y gratificante tanto para hombres como mujeres, y donde se incorporaría una ética del cuidado, es decir, el dispositivo normativo del uso de anticoncepción.

Los datos de entrevista con ambos participantes muestran que no se usa el condón, hay una negación a utilizar métodos anticonceptivos a pesar de tener el conocimiento y acceso a ellos. Las razones para no usarlos en el caso de Andrés: "no se siente igual", para Javier: "ella me dijo que era irregular, que su ginecólogo le dijo que 'quizá no se podría embarazar', dejamos de usar el condón porque en algunas ocasiones teníamos relaciones sexuales 'y no pasaba nada', nos confiamos, hasta que un día pasó... tuvo un retraso, y todavía pensamos... 'no pasa nada', pero pasó... compramos una prueba de embarazo y salió positiva... ¡no lo podíamos creer!”. Esto evidencia que los jóvenes manejan a nivel discursivo el conocimiento y acceso al uso de la anticoncepción, lo cual se consideraría correcto y adecuado sobre las prácticas reproductivas y anticonceptivas, pero que no se pone en práctica. Los datos coinciden con los de Facundo y Vásquez (2008) en la investigación que llevaron a cabo con jóvenes colombianos, señalando que a pesar de la incorporación discursiva de los derechos sexuales y de la retórica de la responsabilidad compartida a través de la proliferación de programas de prevención y planificación, las relaciones sexuales sin uso de anticoncepción en los jóvenes son bastante frecuentes. Contrariamente a la afirmación que se encuentra en la literatura especializada, que señala que el menor uso de métodos anticonceptivos está directamente relacionado con un menor grado de escolaridad o de información al respecto, lo que narran los entrevistados es que generalmente tienen relaciones sexuales sin el uso de métodos anticonceptivos. 


\subsection{Relación afectiva con la pareja y uso de anticoncepción}

Se pudieron identificar dos prácticas de sexualidad, las que se establecen en encuentros ocasionales, generalmente saliendo de fiestas o reuniones donde media el consumo de alcohol y donde los acuerdos con la pareja son implícitos, ya que son con amigas o chicas que conocen en ese momento y donde no se establece una relación formal. Y las relaciones que establecen con una pareja formal "su novia", en este tipo de relación, llegan a postergar el encuentro sexual, establecen relaciones de "respeto" donde no median relaciones sexuales al inicio de la relación de pareja. Y cuando negocian tener vida sexual, al principio llegan a usar anticoncepción, dejando de usarla en el curso del tiempo. En el caso de Andrés y Javier, aun cuando tenían una relación formal de más de un año con sus respectivas parejas, ninguno de los dos usó anticoncepción: "recuerdo que tuvimos relaciones sexuales en casa de mis padres, no tenía condón, y aunque ella me decía que no, yo le dije 'pues de todos modos nos vamos a casar algún día ¿no?, y la convencí..." (Andrés, 24 años).

El discurso anterior, muestra un ejercicio de poder genérico respecto del deseo y uso de la sexualidad por parte de Andrés respecto de su pareja, pues aun cuando ella dice que no a la relación sexual, el argumento de parte de él es "pues de todos modos nos vamos a casar algún día ¿no?", y reafirma "y la convencí...". La relación consolidada con la "novia", valida el que no se recurra al uso de la anticoncepción, bajo el presupuesto normativo de que se llegarían a casar y por tanto asumir la reproducción, el tener hijos, aun cuando ambos estaban estudiando su licenciatura en la universidad.

Es en los encuentros sexuales con parejas informales, donde los entrevistados argumentan que el uso del condón es indispensable, pues no conocen a la pareja, o saben que ha tenido varios compañeros sexuales. En el caso de Andrés y Javier mencionaron haber tenido relaciones sexuales eventuales de manera paralela a su relación de pareja, sin utilizar preservativo. En ambos casos señalaron que conocían los riesgos de contagio asociados a estas prácticas y al mismo tiempo resaltan la importancia de cuidarse en este tipo de relaciones, pero no lo hacen. Consideramos que a pesar de argumentar la idea del cuidado del otro, en la práctica no lo incorporan, por lo menos no en la corresponsabilidad en las decisiones reproductivas y anticonceptivas.

\subsection{La noticia del embarazo}

Dado que no se tenía contemplado un embarazo, la noticia viene a trastocar la vida de ambos miembros de la pareja, sus emociones, actividades, compromisos y responsabilidades. Se enfrentan a un proceso complejo de elaboración y toma de decisión respecto a continuar o interrumpir el embarazo, es una elaboración muy compleja, no se da de un momento a otro, les lleva días, semanas, en ocasiones meses, llevándolos a asumir la continuación del mismo. Una vez que deciden que continuarán con el embarazo, se incorpora la posibilidad de ser padres y se continúa con el proceso de acompañamiento de la pareja. Esto representa el que comuniquen la noticia, enfrentar los cuestionamientos y críticas, pues la expectativa de sus respectivas familias era que terminaran sus estudios universitarios.

La respuesta de las familias es de apoyo para que el hijo pueda continuar con sus estudios universitarios; en ese sentido, ofrecen el que la pareja se vaya a vivir con ellos -en condición de alguien que vino a trastocar la vida y trayectoria de su hijo-, no visualizando la participación de él. En los sectores medios, cuando el hijo asume la responsabilidad del embarazo a partir del tipo de relación afectiva con la pareja, donde se contempla la posibilidad de que sea "la madre de sus hijos", se incorpora la posibilidad de ser padre, de responsabilizarse por sus hechos; sin embargo, su vida nunca será igual.

\subsection{Identidad paterna en estudiantes universitarios}

El proceso de construcción de identidad como padre en estudiantes universitarios se complica, ya que requiere un ejercicio constante de articulación de identidades (estudiante, pareja, padre), por un lado; y continuar como estudiantes, cumplir con las actividades que requiere la escuela, dedicar tiempo al estudio, elaborar trabajos individuales o por equipo, por otro.

Respecto a las vivencias asociadas a la paternidad, la mayoría de los jóvenes se sienten incompetentes y poco preparados, no solo en el terreno afectivo en la relación que van construyendo con su hijo o hija, sino ante las múltiples responsabilidades que el ser padre conlleva. 
La relación de pareja cambia, se reestructura, pues ahora ya no son novios, son jóvenes que están viviendo un proceso de embarazo. En el caso de los entrevistados, se llevaron a vivir a la pareja a casa de los padres de ellos, lo cual implicaba estar constantemente bajo la mirada de los padres de él; esto lleva en ocasiones a dilemas y conflictos entre la pareja, además de continuar con el proceso de embarazo. Y cuando nace el bebé, si bien genera emoción, también un replanteamiento de vida, apareciendo en muchas ocasiones la pregunta “¿qué vamos a hacer?”, pues surge la preocupación por la provisión económica, aun cuando los padres de ambos los estén apoyando.

Ser padre implica un cambio de identidad en los hombres, va asociado a la responsabilidad y al compromiso. La transición a la paternidad es vista como una responsabilidad mayor, donde ya no solo piensan en ellos sino en la pareja, el hijo o la hija, el tiempo que les dedicarán, el cumplimiento de responsabilidades económicas. La paternidad constituye una experiencia importante en la vida, pero la manera cómo se vive cuando son estudiantes universitarios se complica sobremanera. Como señalan Gallardo y cols. (2006), la paternidad es interpretada por los varones en general como un conflicto que los limita, en tanto implica profundas modificaciones en sus proyectos de vida. Dichos cambios devienen de las responsabilidades que se asocian a la conformación y desarrollo de una familia incluyendo la proveeduría económica. Aunado a lo anterior, habría que decir que, en el caso de los jóvenes universitarios, el ser estudiantes e incorporar la vida laboral para cubrir su papel de proveedores se vuelve aun más complejo, pues existe poco apoyo de las instituciones.

El significado que los varones jóvenes dan a la paternidad varía. Algunos piensan en nuevos compromisos y responsabilidades, más años de lucha, de trabajo y de esfuerzo; en otros, miedo, alegría o preocupación, pues se requiere un involucramiento y participación cercana y afectiva con la pareja y el hijo o hija en la vida diaria, mayor compromiso en la crianza y la participación en las actividades domésticas, comunicación, tolerancia y flexibilidad para aceptar los cambios en su trayectoria, haciendo esfuerzos por articular las diferentes identidades: como estudiante, pareja, padre, hijo o trabajador (Salguero 2013).

Ser un buen padre se vuelve una aspiración en los jóvenes entrevistados, para lo cual se hace imperiosa y necesaria una relación de pareja estable, afectiva y comprometida. Una relación de pareja buena y estable permitiría, entre otras cosas, establecer con el hijo un mayor compromiso, estar presente y distribuir roles y tareas de manera compartida y equilibrada con la pareja. Se piensa la paternidad como una experiencia compartida y de aprendizaje, donde lo afectivo está presente.

Si bien, durante los últimos años, se ha abierto el debate en torno a las nuevas formas que ha adoptado la masculinidad en países como México, planteándose que algunos hombres participan en el trabajo doméstico, se involucran en el cuidado de los hijos y expresan más sus sentimientos y emociones. No es algo generalizado, pues se presentan dichos comportamientos más en sectores urbanos, nivel socioeconómico medio, y en jóvenes con escolaridad. Estos cambios no obedecen a la voluntad individual de los varones, sino a complejos procesos psicosociales que involucran estructuras institucionales, a la presión de las mujeres en muchos casos, de manera que se posibilita la emergencia de nuevos referentes sociales de lo masculino, del ser hombre (Salguero y Pérez 2011b). De acuerdo con Bourdieu (1999), la reproducción y mantenimiento de la estructura de género se hace posible a través de las instituciones sociales y estructuras normativas, pero los agentes también desarrollan grados diversos de compromiso con la posición que ocupan asumiendo posturas como la negociación de los discursos y las prácticas de género.

Nos encontramos múltiples identidades masculinas que derivan tanto de las rutas que haya seguido el individuo en su biografía personal, como del grupo de pertenencia y que se modifican a lo largo de la vida. Como señala Connell (2000), las identidades masculinas son construidas históricamente, pero también son constantemente reconfiguradas en las prácticas de género de cada día. Se trata además de identidades que se transforman generacionalmente, pues con la emergencia de nuevas condiciones sociales se deslegitiman ciertas prácticas y se instauran otras. Por ello, el contacto de los jóvenes con nuevas ideas, valores y relaciones con frecuencia se traduce en nuevos modelos de realidad que pueden facilitar el cambio en los patrones de género, como es la participación en las labores domésticas y en la crianza afectiva en la generación de padres jóvenes.

\section{Discusión}


La formación universitaria tiene implicaciones importantes en la formación y trayectoria personal, involucrándose como personas en un proceso continuo de cambio y desarrollo en la construcción de sus identidades. De ahí que sea difícil afirmar que los jóvenes responden como sujetos pasivos ante la estructura institucional, más bien hay una apropiación activa de los patrones y formas de relación presentes en las instituciones educativas, y también distintos mecanismos de resistencia y negociación que los jóvenes utilizan. Es en los escenarios sociales, en términos de Goffman (1979), donde se establecen límites y posibilidades para los individuos, en la medida que se ofrecen mundos de significado con los cuales configuran determinadas visiones de su realidad, orientan sus prácticas cotidianas y establecen posibilidades de relación personal e institucional. Un caso particular es la vivencia de la sexualidad y la reproducción, pues a pesar de que la normatividad institucional señala la prohibición, y lo que interesa es la conclusión del ciclo escolarizado en los tiempos especificados, varios estudiantes estructuran trayectorias distintas a las marcadas por la institución familiar y escolar.

Si bien, el motivo de reflexión es el de los jóvenes universitarios, se sigue requiriendo una mirada amplia para poder documentar la complejidad que representa la vivencia y significado de la sexualidad, la reproducción, la paternidad, ya que trastoca la trayectoria de vida, donde la expectativa contempla la terminación de los estudios universitarios, pero no el ser padre.

Ser padre cuando al mismo tiempo se tiene que ser estudiante, pareja e hijo, resulta de lo más complicado para los jóvenes. Implica un despliegue de identidad en diferentes contextos, lo cual requiere un reordenamiento de vida. Volviéndose una necesidad constante el negociar con la pareja esa nueva forma de vida, como pareja y como padre, y si viven en la casa de sus propios padres, negociar la nueva identidad como hijo.

En esta reconfiguración de vida, se incorpora como expectativa el ser buenos padres, hacer lo mejor que puedan su papel y cumplir con las obligaciones para con sus hijos. Aun cuando los padres jóvenes siguen teniendo presente la solvencia económica y la proveeduría, también se interesan por la relación y el tiempo que puedan pasar con sus hijos, participar activamente en la educación y establecer una relación emocional. No obstante, también aparece en el discurso de los jóvenes la idea de concluir su carrera universitaria, conseguir un empleo y obtener ingresos económicos que les permita cumplir con su papel de proveedores económicos.

El proceso de articulación de identidades en los jóvenes universitarios se posibilita en gran medida a través de las redes sociales de apoyo, en muchos casos las familias resultan fundamentales para que sus hijos estudiantes universitarios/parejas/padres puedan dar continuidad a sus vidas y superen algunos de los problemas que se presentan. Familias cooperativas e interesadas por los estudios, así como amigos que estén estudiando o hayan concluido sus estudios, proporcionan apoyos emocionales, ejemplos, experiencias y expectativas que ayudaran al estudiante a desenvolverse mejor y a tener mayor dedicación cuando se presentan problemas o resulte difícil continuar con su vida académica. Por el contrario, familias distantes o poco interesadas en la problemática del hijo, o amistades que no incentiven la continuidad de los estudios, tendrán un efecto negativo en las expectativas, involucramiento y continuidad académica del joven (Feldman 2008).

Quiénes logran continuar con la trayectoria académica que requiere la universidad y al mismo tiempo ejercer su paternidad requiere ser documentado a través de la investigación, dar cuenta de la historia de vida, las diversas maneras en las que logran articular sus identidades en diferentes contextos como la familia de origen, la escuela, la relación de pareja, la paternidad. Identificar las redes de apoyo y en general los procesos que han permitido que jóvenes universitarios puedan continuar con sus estudios ejerciendo su paternidad.

El ámbito universitario tiene una influencia importante para reforzar o modificar sus concepciones respecto a los modelos de género, sexualidad, reproducción, ética del cuidado. Desde carreras inscritas en las Ciencias Sociales, se plantean posibilidades de reflexión crítica; no obstante, sigue siendo necesario analizar y generar espacios de análisis en los ámbitos universitarios para los estudiantes, pues a pesar de considerarse adultos, en muchos casos no se incorpora el dispositivo sobre el cuidado del otro, en las prácticas de sexualidad no se recurre al uso de la anticoncepción y las implicaciones que de ello se derivan en su trayectoria de vida. 


\section{Notas}

1. Este trabajo se ha realizado en el marco del Proyecto de investigación I+D Laboratorio lberoamericano para el Estudio Sociohistórico de las Sexualidades, financiado por el Ministerio de Economía y Competitividad (FEM2011-27295), y del Proyecto (PAPIT RN306813) financiado por la Universidad Nacional Autónoma de México.

\section{Bibliografía}

Amuchástegui, Ana

2001 Virginidad e iniciación sexual, experiencias y significados. México, Edamex.

2007 "Ética, deseo y masculinidad: la difícil relación entre lo sexual y lo reproductivo", en Ana Amuchástegui e Ivonne Szasz (coords.), Sucede que me canso de ser hombre... Relatos y reflexiones sobre hombres y masculinidades en México. México D. F., El Colegio de México: 121-140.

Andrade, Sally (y otros)

1987 Métodos cualitativos para la evaluación de programas. Un manual para programas de salud, planificación familiar y servicios sociales. Watertown, MA. U.S.A., The Pathfinder Fundation.

Bourdieu, Pierre

1999 Meditaciones pascalianas. Barcelona, Anagrama.

2000 La dominación masculina. Barcelona, Anagrama.

Brannen, Julia (y Ann Nilsen)

2006 "From Fatherhood to Fathering: Transmission and Change among Brithish Fathers in four generations families", Sociology, n²: 335-352.

Castro, Roberto (y Mario Bronfman)

1999 "Problemas no resueltos en la integración de métodos cualitativos y cuantitativos en la investigación social en salud", en Mario Bronfman y Roberto Castro (coord.), Salud, cambio social y política. Perspectivas desde América Latina. México D. F., EDAMEX: 49-64.

Chant, Sylvia (y Nikki Craske)

2003 Gender in Latin America. London, Latin American Bureau.

Connell, Robert

2000 The Men and the Boys. Los Angeles, Berkeley, University of California Press.

De Jesús, David (y Leticia Cabello)

2011 "Paternidad adolescente y transición a la adultez: una mirada cualitativa en un contexto de marginación social", Iberoforum, no 11: 1-27.

Dória, Elisabete (y otros)

1999 "The family man: Conjugality and fatherhood among middle-class Brazilian men on the 1990s", en Coleta de Oliveira (coord.), Os Homens, esses desconohecidos (Masculinidad e Reproducao). Sao Paulo, Brasil (mimeo).

Instituto Nacional de Salud Pública. Secretaría de Salud 2012 Encuesta Nacional de Salud.

Facundo, Ángela (y Carmen Vásquez)

2008 "Nuevos discursos, viejas prácticas: La participación masculina en materia de anticoncepción en el contexto bogotano". Ponencia presentada en Fazendo Gênero 8 - Corpo, Violência e Poder. Florianópolis, 
de 25 a 28 de agosto de 2008: Contracepción; Participación masculina; Trayectorias erótico-afectivas ST 9 - Discursos, políticas e representações no masculino.

Feldman, Lya (y otros)

2008 "Relaciones entre estrés académico, apoyo social, salud mental y rendimiento académico en estudiantes universitarios venezolanos". Universitas Psychology, $\mathrm{n}^{\circ}$ 2: 739-751.

Fleiz, Clara (y otros)

1999 "Conducta sexual en estudiantes de la ciudad de México". Salud Mental (México D. F.), nº 22: 14-19.

Foucault, Michel

1987a Historia de la sexualidad 1. La voluntad de saber. México D. F., Siglo XXI.

1987b Historia de la sexualidad 3. La inquietud de sí. México D. F., Siglo XXI.

1988 Historia de la sexualidad 2. El uso de los placeres. México D. F., Siglo XXI.

Fuller, Norma

2000 "Significados y prácticas de paternidad entre varones urbanos del Perú", en Norma Fuller (ed.), Paternidades en América Latina. Perú, Pontificia Universidad Católica del Perú, Fondo Editorial: 35-90.

Gallardo, Gonzalo (y otros)

2006 "Paternidad: representaciones sociales en jóvenes varones heterosexuales universitarios sin hijos", Psykhe, n²: 105-116.

Giddens, Anthony

1998 La transformación de la intimidad. Madrid, Ediciones Cátedra.

Goffman, Erving (y Fernando Santos Fontenla).

1979 Relaciones en público: Microestudios del orden público. Madrid, Alianza.

González Garza, Carlos (y otros)

2005 "Perfil del comportamiento sexual en adolescentes mexicanos de 12 a 19 años de edad. Resultados de la ENSA 2000", Salud Pública de México (México D. F.), nº 3: 209-218.

Gutmann, Matthew

2000 Ser hombre de verdad en la ciudad de México. Ni macho ni mandilón. México D. F., El Colegio de México.

Haces, María de los Ángeles

2006 "La vivencia de la paternidad en el valle de Chalco", en Juan Guillermo Figueroa y otros (coord.), Ser padres, esposos e hijos: prácticas y valoraciones de varones mexicanos. México D. F., El Colegio de México: 121-155.

Hoghughi, Masud (y Nicholas Long)

2004 "Key Concepts. Parenting-An Introduction”, en Masud Hoghughi and Nicholas Long (coord.), Handbook of Parenting. New Delhi, SAGE: 1-18.

lbáñez, Berenice

1998 "Conducta sexual y embarazo en adolescentes de Tijuana, B. C", La Psicología Social en México (México D. F.), nº 7: 288-293.

Jiménez, $M^{a}$ Lucero

2001 La reproducción de los varones en México. El entorno sexual de la misma, estudios de casos. Tesis Doctorado en Sociología. FCPyS, UNAM. México D. F.

Kaufman, Michael

1994 "Men, feminism, and men's contradictory experiences of power", en Harry Brod and Michael Kaufman (eds.), Theorizing Maculinities. Thousand Oaks, CA: SAGE: 119-141.

Lagarde, Marcela 
1993 Los cautiverios de las mujeres: madresposas, monjas, putas, presas y locas. México, UNAM.

Lamas, Marta

1997 "Usos, dificultades y posibilidades de la categoría "género". Diferencias de Idioma, Analogías y Confusiones Conceptuales", en Marta Lamas (coord.), El Género: la construcción cultural de la diferencia sexual. México D. F., UNAM/Porrúa: 327-366.

Menkes, Catherine

2004 "Embarazo y fecundidad adolescente en México", en Fernando Lozano (coord.), El amanecer del siglo y la población mexicana. VI Reunión Nacional de Investigación Demográfica en México, México, Universidad Nacional Autónoma de México. Centro Regional de Investigaciones Multidisciplinarias, Sociedad Mexicana de Demografía: 109-129.

Nava, Regina

1996 Los hombres como padres en el Distrito Federal a principios de los noventa. Tesis Maestría en Sociología. FCPyS UNAM. México D. F.

Núñez, Guillermo

2007 'Vínculo de pareja y hombría: 'Atender y mantener' en adultos mayores del Río Sonora, México”, en Ana Amuchástegui e Ivonne Szasz (coords.), Sucede que me canso de ser hombre... Relatos y reflexiones sobre hombres y masculinidades en México. México D. F., El Colegio de México: 141-184.

Rojas, Olga Lorena

2000 La paternidad y la vida familiar en la ciudad de México, un acercamiento cualitativo al papel desempeñado por los varones en los ámbitos reproductivo y doméstico. Tesis Doctorado en Estudios de Población. El Colegio de México, A. C., Centro de Estudios Demográficos y de Desarrollo Urbano. México D. F.

Rubin, Gayle

1997 "El tráfico de mujeres: notas sobre la "Economía Política" del sexo", en Marta Lamas (comp.), El género: La construcción cultural de la diferencia sexual. México D. F., Porrúa: 35-96.

Salguero Alejandra

2002 Significado y vivencia de la paternidad en el proyecto de vida de los varones. Tesis de Doctorado en Sociología. Facultad de Ciencias Políticas y Sociales. Universidad Nacional Autónoma de México.

2013 "Masculinidad como configuración dinámica de identidades", en Juan Carlos Ramírez y José Carlos Cervantes (coord.), Los hombres en México. Veredas recorridas y por andar. Una mirada a los estudios de género de los hombres, las masculinidades. México, Universidad de Guadalajara. CUCEA, AMEGH: 37-52.

Salguero, Alejandra (y Gilberto Pérez)

2008 "La paternidad en los varones: Una búsqueda de identidad en un terreno desconocido. Algunos dilemas, conflictos y tensiones", Revista Internacional de estudios sobre masculinidades, $\mathrm{n}^{\circ}$ 4: 1-18

2011a Dilemas y conflictos en el ejercicio de la maternidad y la paternidad. México D. F., UNAM, FESiztcala.

2011b La paternidad en el cruce de perspectivas: El discurso reflexivo de padres y madres, GénEros, (México D. F.), nº 9: 35-56.

Salles, Vania (y Roberto Tuirán)

1998 "Cambios demográficos y socioculturales: familias contemporáneas en México", en Beatriz Schmukler, Familia y relaciones de género en transformación. México D. F., Edamex: 83-126.

Szasz, Ivonne

1997 "La sexualidad de los adolescentes mexicanos", Revista adolescencia (México D. F.), n 4: 5-15.

Seidler, Victor 
2000 La Sinrazón Masculina. Masculinidad y teoría social. México D. F., UNAM, Porrúa, Paidós.

Stern, Claudio

2004 "Vulnerabilidad social y embarazo adolescente en México", Papeles de población (México D. F.) nº 39: 129-158.

Testa, Julio (y Pablo Sánchez)

2003 "El enfoque de las trayectorias educativas y laborales como una mirada complementaria en el tratamiento de la problemática universitaria". Ponencia presentada en el Congreso latinoamericano de educación superior en el siglo XXI, Universidad Nacional de San Luis, Argentina, 18-20 de septiembre.

Torrado, S.

2006 Familia y diferenciación social. Cuestiones de método. Buenos Aires, Eudeba.

Vendrell, Joan

2010 "Masculinidad y paternidad. La apropiación de la capacidad reproductiva de las mujeres en el origen de la dominación masculina", en Diana Córdoba y otros (coord.), Sexualidad de los varones. Anticoncepción, gestación y paternidad. México D. F., UNAM, FESI: 1-13.

Viveros, Mara (y William Cañón)

1997 “Pa'bravo... yo soy candela, palo y piedra. Los quibdoseños”, en Teresa Valdéz y José Olavarría (eds.), Masculinidad/es. Poder y crisis. Isis. Chile, FLACSO: 125-138.

Wainerman, C.

2003 Familia, Trabajo y Género. Un mundo de nuevas relaciones. Buenos Aires, Fondo de Cultura Económica-UNICEF.

Walzer, Susan

2004 "Encountering oppositions. A review of scholarship about motherhood", en Marilyn Coleman y Lawrence H. Ganong (eds.), Handbook of contemporary families. Considering the past, contemplating the future. Thosands Oaks, Ca, Sage Publications: 209-223. 